\title{
To Whom Are We Talking?
}

As the major worldwide professional organization in the domain of humancomputer interaction, SIGCHI has a diverse audience and a large portfolio of questions and messages. Our main goal is to support the international community of HCI experts in its professional life.

Consequently, the foremost activities of our organization consist of supporting members to meet and to communicate in several ways:

- physically, during conferences, located either where the crowds are or set between multiple local communities. Volunteers such as vice president for conferences Scooter Morris, adjunct chair for specialized conferences Philippe Palanque, members of our conference management committee and a growing list of steering committees, in collaboration with the ACM staff, work on site selections and with local HCI groups; compose conference committees and invite reviewers; and stimulate our members and other members of the HCI community to submit and to attend.

- archivally, through our peerreviewed journal; through the evangelizing and more colorful tone of Interactions, and by storing the growing body of knowledge in the ACM Digital Library. Our vice president for publications Dan Olsen, along with the editors and the papers chairs of our many conferences, are the basis of this volunteer work.
- in the cloud, through websites, mailing lists, blogs, Twitter accounts, and other social media, where executive vice president Elizabeth Churchill, vice president for operations Fred Sampson, and vice chair for social media Patrick Kelley are all involved.

Since our members and other HCI professionals are now spread out over more parts of the globe, there is a growing need for reaching out to other HCI-related groups and for developing new local societies. Vice president for chapters Tuomo Kujala, IFIP liaison John Karat, and adjunct chair for the developing world Zhengjie Liu are continuously building networks and working on new developments.

Like any professional society, SIGCHI aims at eternal life, though in reality our members are quite mortal. So there is a structural need for recruiting new and young members. Students in the field must be educated, and curricula must be specified, a continuing task for our adjunct chair for education Jenny Preece, who works with Elizabeth Churchill and parttime researcher Anne Bowser. Apart from building a detailed global map of educational practices and education needs, they are aiming to find a way to evangelize to educational authorities worldwide for HCI education quality and relevant coverage.

As the major professional society for HCI, we have a message for society at large. Interactive systems should fit users' abilities and understandings, as well as their needs. This requires involvement in standards, in developing quality criteria for documentation, and in making users and members of industry aware of what we do. These are areas in which we can bolster our efforts. We could do with more expertise and creative volunteers to help us better coordinate, get a stronger and more consistent voice, and develop enough critical mass to be taken seriously by industry - perhaps even be approached by them.

SIGCHI is not the only professional society in the world that focuses on HCI. We need not compete with the others, however, to serve our membership and the international HCI profession. Instead, we want to communicate with them and, wherever feasible, to collaborate. All of our communication tasks sketched here are intended to serve HCI worldwide. So, wherever you are, if you are an HCI person, make sure you find a SIGCHI group around you and, please, talk to us. If you have enough time and energy, consider contributing in any way that fits your context. And know that non-SIGCHI members can join our communities at no charge at http://www.sigchi. org/communities. If you want to vote, you should become a member, but that seems reasonable, right? - Gerrit C. van der Veer President, ACM SIGCHI 